\title{
Ubenimex enhances the radiosensitivity of renal cell carcinoma cells by inducing autophagic cell death
}

\author{
SHUAI LIU $^{1 *}$, XIAOQING WANG ${ }^{1 *}$, JIAJU LU $^{1}$, LIPING HAN $^{2}$, YONGFEI ZHANG ${ }^{3}$, \\ ZHENG LIU $^{1}$, SENTAI DING ${ }^{1}$, ZHAO LIU $^{1}$, DONGBIN BI ${ }^{1}$ and ZHIHONG NIU ${ }^{1}$ \\ ${ }^{1}$ Department of Urology, Shandong Provincial Hospital Affiliated to Shandong University, Jinan, Shandong 250021; \\ ${ }^{2}$ Department of Neurology, Shandong Police Hospital, Jinan, Shandong 250021; ${ }^{3}$ Department of Dermatology, \\ Shandong University, Jinan, Shandong 250000, P.R. China
}

Received April 8, 2015; Accepted June 3, 2016

DOI: $10.3892 / 01.2016 .5036$

\begin{abstract}
Renal cell carcinoma (RCC) is resistant to standard radiotherapy. Ubenimex, an aminopeptidase $\mathrm{N}$ inhibitor, is widely used as an adjunct therapy after surgery to enhance the function of immunocompetent cells and confer antitumor effects. Our previous study demonstrated that ubenimex induces autophagic cell death in RCC cells. Recently, the molecular mechanism of autophagy induction has been associated with radiosensitivity in RCC cells. In the present study, the ability of ubenimex to enhance RCC cell sensitivity to radiation via the induction of autophagic cell death was determined, and the mechanism of action of this effect was investigated. The 786-O and OS-RC-2 human RCC cell lines were treated with $0.5 \mathrm{mg} / \mathrm{ml}$ ubenimex and different doses of irradiation (IR). The cell viability was measured using a colony-formation assay and flow cytometry. Acridine orange (AO)-ethidium bromide (EB) staining was assessed by fluorescence microscopy as an indicator of autophagic cell death. Protein expression was assessed by western blotting. Autophagosomes were evaluated using transmission electron microscopy. RCC cells were used to evaluate the sensitivity to radiation using clonogenic survival and lactate dehydrogenase assays. Furthermore, these parameters were also tested at physiological oxygen levels. The AO-EB staining and flow cytometry of the OS-RC-2 cells indicated that the combined treatment significantly enhanced autophagic cell death compared with ubenimex or IR alone. Therefore, treatment with ubenimex did not significantly alter cell cycle progression but increased cell death when combined with radiation. An Akt agonist could significantly
\end{abstract}

Correspondence to: Mr. Zhihong Niu, Department of Urology, Shandong Provincial Hospital Affiliated to Shandong University, 324 Jingwu Street, Jinan, Shandong 250021, P.R. China

E-mail:nzh1789@163.com

*Contributed equally

Key words: ubenimex, radiosensitivity, RCC, autophagic cell death, Akt weaken this effect, indicating that ubenimex may act as an Akt inhibitor. Furthermore, the western blot analysis indicated that the combined treatment inhibited the Akt signaling pathway compared with ubenimex treatment or IR alone. Ubenimex may enhance RCC cell sensitivity to radiation by inducing cell autophagy. This induction changes the role of autophagy from protective to lethal in vitro, and this switch is associated with the inhibition of the Akt signaling pathway.

\section{Introduction}

Renal cell carcinoma (RCC) is the most common type of renal cancer, and more than half of RCC patients are identified at the advanced stages and present with a local or systematic metastasis, resulting in a poor prognosis (1). Patients presenting with advanced RCC have a poor prognosis due to the chemo- and radioresistance of this disease $(2,3)$. Specifically, tumor cells respond to radiation via multiple growth arrests and a form of protective autophagy (4-8). Therefore, radiotherapy is rarely used to treat RCC in the clinic (9), and novel anti-tumor agents to reverse the radiosensitivity of RCC are urgently required.

In cell biology, autophagy or autophagocytosis, which is defined as 'self-eating', is a catabolic process that involves the degradation of the components of a cell via the lysosomal machinery $(10,11)$. The important role of autophagy in cancer therapy has become increasingly apparent. Under normal conditions, autophagy is a mechanism that ensures the turnover of proteins and elimination of damaged organelles to maintain cell homeostasis (12). By contrast, it functions as an adaptive cell response under pathological conditions, allowing the cell to survive bio-energetic stress (13). However, extensive or persistent autophagy also results in cell death (14). Therefore, autophagy is a decisive factor between cell death and survival, and can be either protective or lead to autophagic cell death. Notably, the induction of autophagy enhances the effects of radiation (15-22). However, the contribution of autophagy to radiation efficacy remains unclear, particularly in RCC.

Recently, the role of autophagy as an alternative cell death mechanism has been a topic of debate. Ongoing studies are attempting to define optimal strategies to modulate autophagy forcancer prevention and therapy, as well as to exploit autophagy as a target for anticancer drug discovery (23). The activation of 
the phosphoinositide 3-kinase (PI3K)/Akt signaling pathway, a well-known method of inhibiting apoptosis, also inhibits autophagy (24). Additionally, increasing evidence indicates that DNA damage induces autophagy (20-22), and that inhibiting Akt enhances the cytotoxicity of DNA-damaging agents in several cancer cells (25). In addition, an Akt inhibitor could cause a switch from protective autophagy to autophagic cell death. This switch enhanced sorafenib resistance and cell proliferation in RCC cells $(8,26,27)$. However, similar studies on $\mathrm{RCC}$ radiotherapy are rare.

Ubenimex is widely used as an adjunct therapy after surgery to enhance the function of immunocompetent cells and confer antitumor effects. Ubenimex has been widely used as a therapy for leukemia, non-small cell lung cancer, gastric cancer and cervical cancer (28-30). Our previous study demonstrated that ubenimex induces the autophagic cell death of RCC cells (31). We propose that autophagic cell death induced by ubenimex may be associated with an autophagy-related signaling pathway such as the Akt pathway. Therefore, the objective of the present study was to determine the ability of ubenimex to enhance RCC cell sensitivity to radiation by inducing autophagic cell death and changing the role of autophagy from protective to harmful. We also sought to determine the association of this effect with Akt.

\section{Materials and methods}

Cell culture. The 786-O and OS-RC-2 RCC cell lines were purchased from the cell bank of the Chinese Academy of Sciences (Beijing, China). The cells were maintained in RPMI-1640 medium (HyClone; GE Healthcare Life Sciences, Logan, UT, USA) supplemented with penicillin, streptomycin and $10 \%$ fetal bovine serum (Biological Industries, Cromwell, $\mathrm{CT}$, USA). The cells were incubated at $37^{\circ} \mathrm{C}$ in a humidified atmosphere containing $5 \% \mathrm{CO}_{2}$.

Irradiation (IR). The cells were irradiated with $\gamma$-rays from a ${ }^{137} \mathrm{Cs}$ irradiator (GSR D1; Gamma-Service Medical GmbH, Leipzig, Germany) at a dose rate of $1.7 \mathrm{~Gy} / \mathrm{min}$. For IR under hypoxic conditions, the cells were sealed inside a hypoxia chamber in purpose-built airtight boxes and then transported to the irradiator. Dosimetry was performed using EBT2 film (Ashland Inc., Covington, KY, USA) irradiated in the position of cells. The exposed EBT2 film strips were scanned, and the optical density values were corrected as recommended by the manufacturer and converted to a dose using a calibration curve obtained from previously scanned film strips irradiated with a range of known doses using ${ }^{60} \mathrm{Co} \gamma$-rays.

Cell viability and colony-formation assay. The cells were seeded in 96-well plates, treated at different IR doses $(0,2,4$ and $6 \mathrm{~Gy})$ or for different times $(0,12,24$ and $36 \mathrm{~h})$, and then allowed to grow for $72 \mathrm{~h}$. Dimethyl sulfoxide was used as the vehicle. The cell viability was observed using the trypan blue dye-exclusion assay, and the viable cells were counted using a hemocytometer. To determine the long-term effects of treatment on cell colony formation, the cells were seeded in 6 -well plates at a density of 2,000 cells/well and treated with different doses $(0,2,4$ and 6 Gy) of IR. After rinsing with fresh medium, the cells were allowed to grow for 14 days to form colonies, which were stained with crystal violet $(0.5 \% \mathrm{w} / \mathrm{v})$, photographed with a scanner and counted.

Western blot analysis. To determine the expression of microtubule-associated protein 1A/1B-light chain 3B (LC3B), Akt and phosphorylated (p)-Akt, the proteins were extracted from cells or tissues by resuspension in radioimmunoprecipitation assay buffer. The samples were centrifuged at $12,000 \mathrm{x} \mathrm{g}$ at $4^{\circ} \mathrm{C}$ for $30 \mathrm{~min}$, and the supernatants were recovered for analysis. The protein concentrations were determined using the Bradford protein method and a bicinchoninic acid protein assay kit (Sigma-Aldrich, St. Louis, MO, USA). The proteins $(40 \mu \mathrm{g})$ were electrophoresed on a pre-cast Bis-Tris polyacrylamide gel (8\%) and then transferred to a polyvinylidene difluoride membrane. The membranes were blotted with rabbit anti-p-Akt (1:1,000; catalog no. 9271S; Cell Signaling Technology, Inc., Danvers, MA, USA), rabbit anti-LC3B (1:1,000; catalog no. L7543; Sigma-Aldrich) rabbit anti-Akt (1:1,000; catalog no. 9272; Cell Signaling Technology, Inc.) or mouse anti-glyceraldehyde 3-phosphate dehydrogenase (1:3,000; catalog no. TA08; ZsBio, Beijing, China), followed by incubation with the corresponding horseradish peroxidase-conjugated secondary antibodies (1:5,000; catalog nos. ZB2306 and ZB2301; ZsBio), overnight at $4^{\circ} \mathrm{C}$. The immunoblots were visualized using enhanced chemiluminescence and ImageQuant LAS 4000 (GE Healthcare Life Sciences).

Lactate dehydrogenase ( $L D H)$ cytotoxicity assay. The levels of LDH release were assessed by determining the extent of cell death irrespective of the type of death. A 200- $\mu 1$ volume of cell suspension in complete medium $\left(5 \times 10^{3}\right.$ cells/well) was dispensed into each well of a 96-well plate. The cells were treated with ubenimex and/or IR at different times. The 96-well plates were centrifuged for $5 \mathrm{~min}$ at $400 \mathrm{x}$ g, and $120 \mu \mathrm{l}$ supernatant from each well was then transferred to a new plate. The plates were incubated at room temperature for 30 min in the dark, and the absorbance was then spectrophotometrically measured at a wavelength of $560 \mathrm{~nm}$.

Electron microscopy (EM). The RCC cells were treated with $0.5 \mathrm{mg} / \mathrm{ml}$ ubenimex for $12 \mathrm{~h}$ and/or irradiated (4 Gy) for $24 \mathrm{~h}$. The cells were then fixed with $3 \%$ glutaraldehyde and $2 \%$ paraformaldehyde in $0.1 \mathrm{M}$ phosphate-buffered saline (PBS) for 30 min, post-fixed with $1 \%$ osmium tetroxide (Absin Bioscience Inc., Shanghai, China) for $1.5 \mathrm{~h}$, washed, stained in $3 \%$ aqueous uranyl acetate (Haoranbio, Nanchang, China) for $1 \mathrm{~h}$, dehydrated in an ascending series of ethanol and acetone, and embedded in Araldite ${ }^{\circledR}$ (Huntsman Advanced Materials LLC, The Woodlands, TX, USA). Ultrathin sections were cut on a Reichert-Jung ultramicrotome (Leica Microsystems, Inc., Buffalo Grove, IL, USA), double stained with $0.3 \%$ lead citrate (Alfa Chemistyry, Stony Brook, NY, USA) and examined on a 1200EX electron microscope (JEOL, Ltd., Tokyo, Japan).

Acridine orange (AO)-ethidium bromide (EB) double staining. The DNA-binding dyes AO and EB are used for the morphological detection of autophagic cell death (32). A cocktail of EB and $\mathrm{AO}(100 \mu \mathrm{g} / \mathrm{ml})$ was prepared in PBS. AO is uptaken by both viable and non-viable cells and emits green fluorescence, whereas EB is uptaken only by non-viable cells and emits red 
fluorescence via intercalation into DNA (33). Following IR, the cells were washed twice with PBS, and fresh medium was added. After a 30-min incubation, the cells were washed again with PBS, stained with $\mathrm{AO} / \mathrm{EB}$ and incubated for $30 \mathrm{~min}$ in the dark. Next, the cells were washed with PBS and analyzed by fluorescence microscopy (34). The percentage of positively stained cells was calculated to determine the death rate (\%), which is the number of cells undergoing programmed cell death per 100 cells. The experiments were repeated thrice.

Annexin V-fluorescein isothiocyanate (FITC)/propidium iodide (PI) staining. The apoptotic cells were quantified (\%) using an annexin V-FITC)/PI kit (Nanjing KeyGen Biotech, Co. Ltd., Nanjing, China) and detected by flow cytometry. The RCC cells were harvested after $12 \mathrm{~h}$ of treatment with ubenimex and/or after $24 \mathrm{~h}$ of IR. Next, the cells were resuspended in binding buffer (10 mmol/1 4-(2-hydroxyethyl)-1-piperazineethanesulfonic acid, $140 \mathrm{mmol} / \mathrm{l} \mathrm{NaCl}$ and $2.5 \mathrm{mmol} / \mathrm{l}$ $\mathrm{CaCl}_{2}, \mathrm{pH}$ 7.4) and incubated with annexin V-FITC/PI in the dark for $15 \mathrm{~min}$. A total of 5,000 cells/sample were analyzed using a FACSCalibur or an EPICS XL flow cytometer (BD Biosciences, Franklin Lakes, NJ, USA). The cells in the early stages of apoptosis stained positive for annexin V-FITC, whereas those in the late stages of apoptosis stained positive for both annexin V-FITC and PI.

Statistical analysis. The data were statistically analyzed with Student's t, $\chi^{2}$ or Fisher's exact tests using SPSS version 19.0 (IBM SPSS, Armonk, NY, USA). P $<0.05$ was considered to indicate a statistical significant difference.

\section{Results}

Pretreatment with ubenimex significantly enhances the toxicity of $I R$ and reduces the survival fraction in a dose-dependent manner compared with IR alone. The viability of cells was determined at different doses of IR (0, 2, 4 and 6 Gy) for $24 \mathrm{~h}$ (Fig. 1A) or different times of IR $(0,12,24,36$ and $48 \mathrm{~h})$ at a dose of 4 Gy (Fig. 1B). The viability of 786-O and OS-RC-2 cells varied with the different treatments. The LDH cytotoxicity assay of OS-RC-2 cells revealed that the number of dead cells positively correlated with the time and dose of IR (Fig. 1C). Ubenimex and IR alone or in combination were used to treat 786-O cells. The combined treatment significantly increased toxicity in 786-O cells compared with ubenimex or IR treatment alone (Fig. 1E). Furthermore, the radiation dose-response survival curves for 786-O cells with or without ubenimex treatment were significantly shifted downward for ubenimex-treated cells (Fig. 1D), indicating that ubenimex increased IR-induced clonogenic cell death in 786-O cells.

Compared with IR alone, pretreatment with ubenimex induced cell death, including autophagic cell death and apoptosis. IR plays a key role in cancer therapy because it directly induces cell death (35). AO-EB staining is a sensitive tool for the estimation of autophagic cell death (32), requiring only a small number of cells. The AO-EB staining of OS-RC-2 cells indicated that the combined treatment of ubenimex and IR significantly enhanced cell death compared with ubenimex or IR treatment alone. Additionally, this combined treatment significantly increased the number of EB-positive cells compared with ubenimex treatment alone. These results suggest that autophagic cell death may be involved in the anti-proliferative effects of the above combined treatment on OS-RC-2 cells (Fig. 2).

Compared with IR alone, pretreatment with ubenimex enhances early apoptosis and autophagic cell death in $O S-R C-2$ cells. Flow cytometry was used to further detect cell death. As represented in Fig. 3, early apoptosis and autophagic cell death in OS-RC-2 cells was measured by flow cytometry with an annexin $\mathrm{V}$ apoptosis detection kit (Fig. 3). The quantitative results revealed that the combined treatment of ubenimex and IR induced significant early apoptosis and autophagic cell death in OS-RC-2 cells compared with treatment with ubenimex or IR alone.

Combined treatment induces type II programmed cell death (autophagic cell death). The ultrastructure of the OS-RC-2 cells for each treatment group was observed by EM photomicrography (Fig. 4A). The combined treatment resulted in a large number of autophagic vacuoles and autolysosomes in the cytoplasm (Fig. 4B). In addition, chromatin condensation or nuclear pyknosis, which are characteristic of apoptosis (36), were not observed in any of the treated cells. To detect the expression of autophagy-related proteins, the lysates from OS-RC-2 cells subjected to different treatments were examined by western blotting (Fig. 3B). The combined treatment of ubenimex and IR increased the expression levels of the LC3B protein, suggesting that the combined treatment induced autophagic cell death in OS-RC-2 cells (Fig. 3B).

Akt signaling pathway is involved in the effect of the combined treatment of ubenimex and IR, changing autophagy from protective to harmful in $O S-R C-2$ cells. Previous studies have demonstrated that the Akt signaling pathway is involved in the regulation of autophagy (26). Specifically, stress appears to activate the Akt signal transduction pathway in tumor cells, which results in protective autophagy $(8,27)$. Therefore, to determine the involvement of the Akt signaling pathway in the effect of the above combined treatment, the levels of protein phosphorylation were detected by western blotting (Fig. 4C). The phosphorylated proteins involved in the Akt signaling pathways were also examined. The combined treatment decreased the phosphorylation of Akt compared with ubenimex treatment or IR alone. As indicated in Figs. 1E and 4, the autophagic cell death induced by radiation, but IR combined with an Akt inhibitor or ubenimex strongly induced autophagic cell death, whereas the addition of an Akt agonist significantly decreased these effects (Fig. 1E).

\section{Discussion}

Radioresistance may be associated with protective autophagy. As previously reported (37), IR treatment induced autophagy in RCC cells. However, it did cause significant cell death compared with ubenimex (32). Akt may be the main mechanism of autophagic cell death, since the ectopic overexpression of Akt partly rescues cancer cells from death when they are stressed (8). Additionally, previous studies have demonstrated 
A

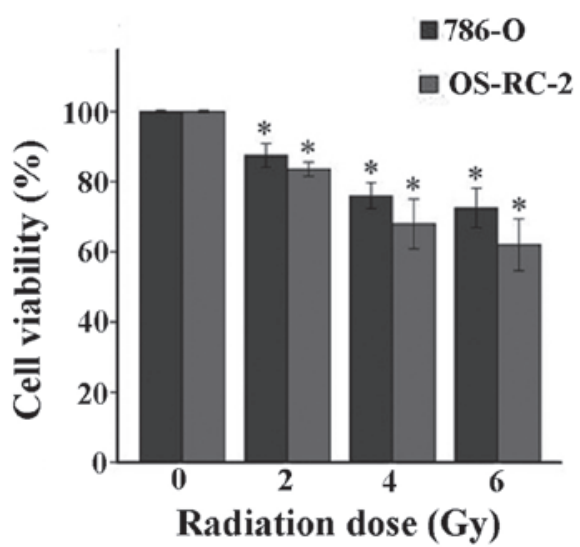

C

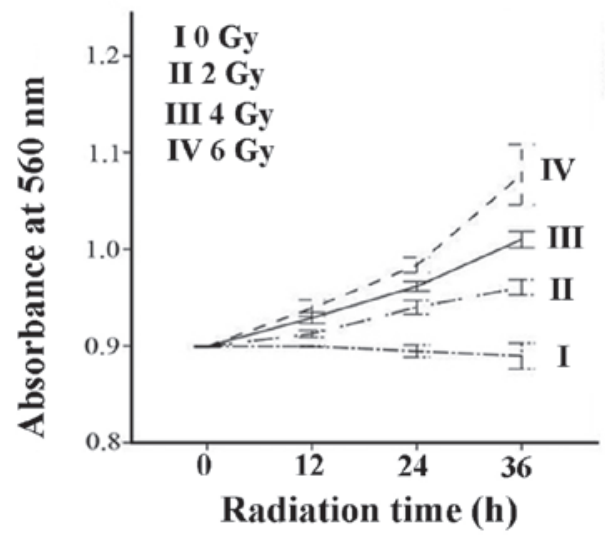

B

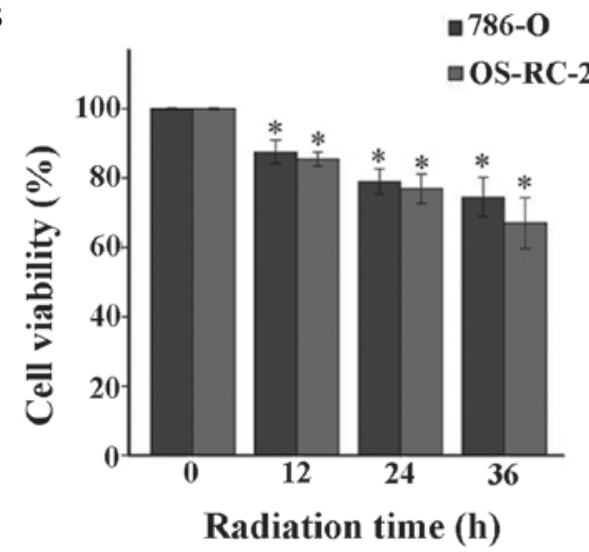

D

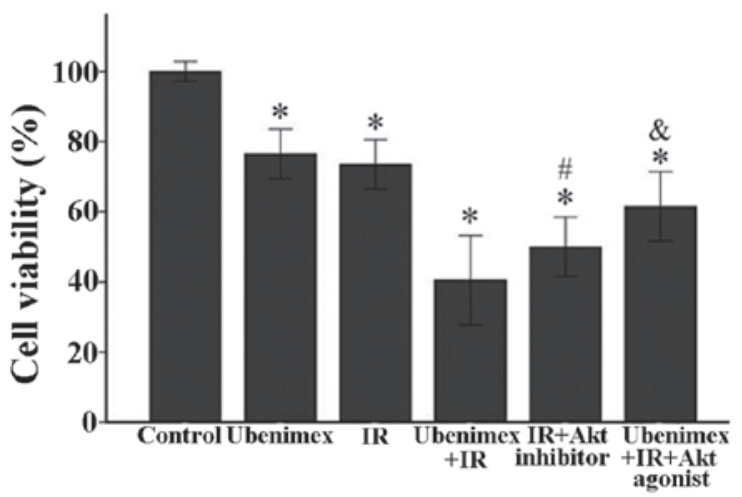

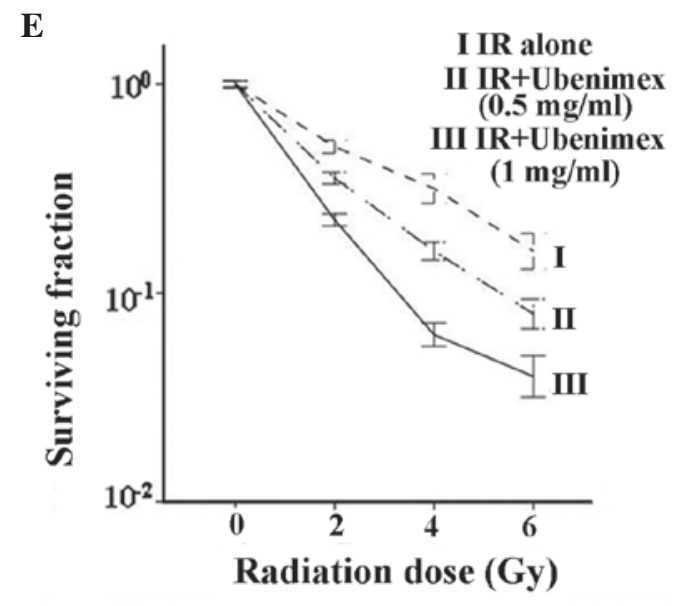

Figure 1. (A) Dose-dependent effects of IR on the viability of RCC cells. Cells were treated with $0,2,4$ or 6 Gy IR for 24 h. *P<0.05, IR vs. control. (B) Time-dependent effects of IR on the viability of RCC cells. Cells were treated for $0,12,24$ or $36 \mathrm{~h}$ with $4 \mathrm{~Gy}$ IR. ${ }^{*} \mathrm{P}<0.05$, IR vs. control. (C) Lactate dehydrogenase cytotoxicity assay was performed at different times and different doses of IR treatment in OS-RC-2 cells. ${ }^{*} \mathrm{P}<0.05$ vs. untreated cells. (D) Cytotoxic effects in 786-O cells treated with IR (4 Gy) and/or ubenimex $(0.5 \mathrm{mg} / \mathrm{ml})$. The IR+Akt inhibitor and IR+ubenimex $+\mathrm{Akt}$ agonist were also tested. ${ }^{*} \mathrm{P}<0.05$ vs. control. ${ }^{\#} \mathrm{P}<0.05$, IR+ubenimex or IR+Akt inhibitor vs. IR treatment. ${ }^{\&} \mathrm{P}<0.05$, IR+ubenimex+Akt agonist vs. IR+ubenimex treatment. (E) Radiation dose-response survival curves of 786-O cells with or without ubenimex. All results are presented as the mean \pm standard deviation from three independent experiments. IR, irradiation; RCC, renal cell carcinoma.

that stress activated the Akt signal transduction pathway in tumor cells and induced protective autophagy, whereas the inhibition of the Akt signaling pathway synergistically killed cancer cells (27). In the present study, the addition of ubenimex or an Akt inhibitor significantly induced autophagic cell death, whereas an Akt agonist reduced this effect. Therefore, it was concluded that IR induces protective autophagy, but the addition of ubenimex or an Akt inhibitor inhibits the Akt signaling pathway, thus switching the role of autophagy from protective to lethal.

Autophagic cell death is a death mechanism that is distinguishable from apoptosis (23). Autophagy could lead to cell death, but in certain cases, such as when faced with chemotherapy, it could be a way for tumor cells to survive. In multiple 
A
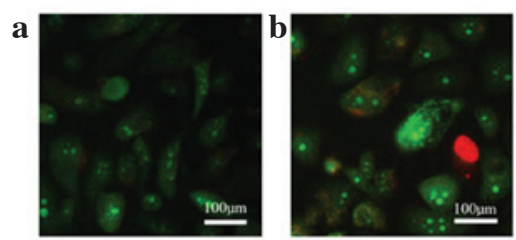

c
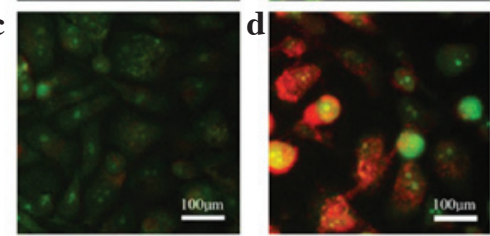

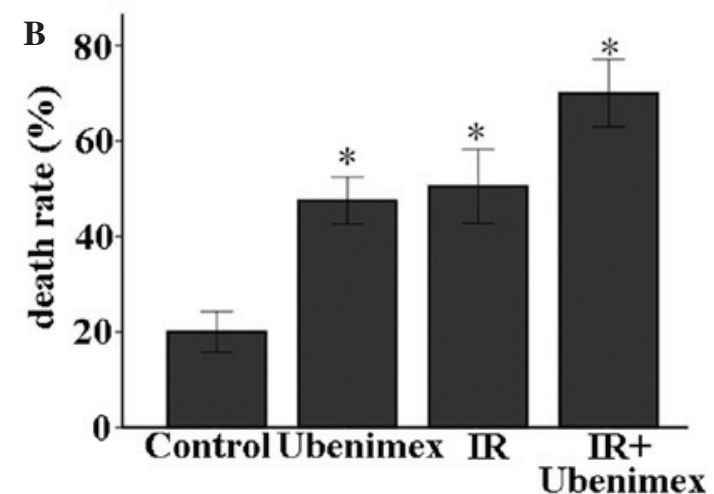

Figure 2. Acridine orange-ethidium bromide staining in OS-RC-2 cells treated with IR (4 Gy) or ubenimex $(0.5 \mathrm{mg} / \mathrm{ml})$ alone or in combination for $24 \mathrm{~h}$. Red fluorescence was used to identify autophagic cell death (32). (Aa) Control; (Ab) Cells treated with ubenimex (0.5 mg/ml) alone; (Ac) Cells treated with IR (4 Gy) alone; (Ad) cells treated with combined ubenimex $(0.5 \mathrm{mg} / \mathrm{ml})$ and IR (4 Gy). Significant cell death was observed for the combined treatment of ubenimex and IR compared with ubenimex or IR alone. The percentage of positively stained cells was calculated and considered to be the death rate (\%), which is the number of cells undergoing programmed cell death per 100 cells. ${ }^{*} \mathrm{P}<0.05$, vs. control. All data are presented as the mean \pm standard deviation from three independent experiments. IR, irradiation.

A
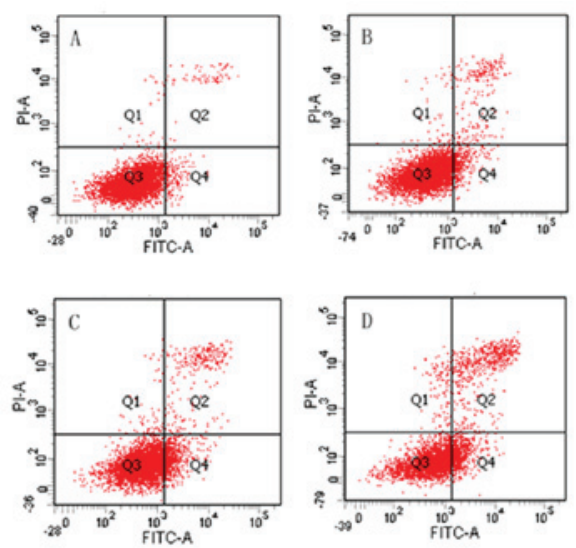

C

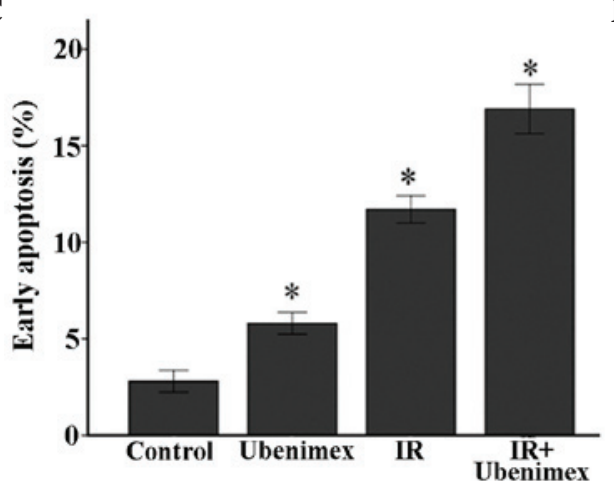

B

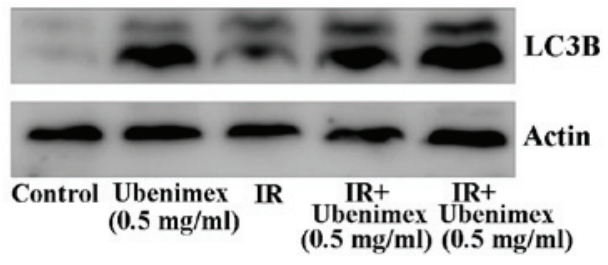

D

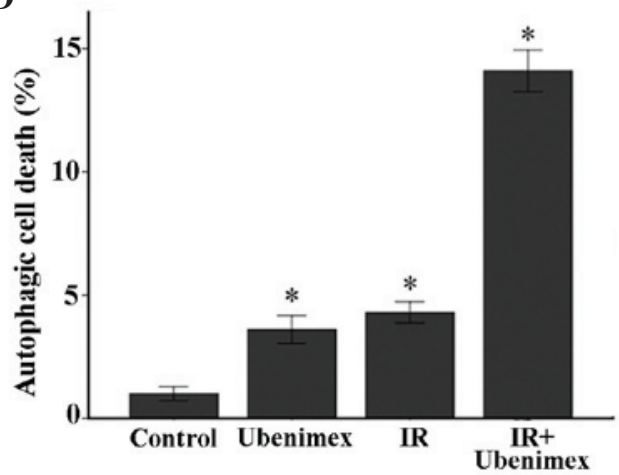

Figure 3. (A) OS-RC-2 cells were treated with IR (4 Gy) or ubenimex $(0.5 \mathrm{mg} / \mathrm{ml})$ alone or in combination for $24 \mathrm{~h}$, and then detected using flow cytometry. (B) Western blotting for microtubule-associated proteins 1A/1B light chain 3B in OS-RC-2 cells. Cells were treated with IR (4 Gy) or ubenimex alone or in combination for $24 \mathrm{~h}$. Actin was used as a loading control. Bars indicate the mean \pm SD from three independent experiments ("P $<0.05)$. (C) Early apoptosis, detected using an annexin $\mathrm{V}$ apoptosis detection kit, was measured using flow cytometry. Cells were treated with IR (4 Gy) and/or ubenimex (0.5 mg/ml). ${ }^{*} \mathrm{P}<0.05$ vs. control. (D) Autophagic cell death. Fluorescein isothiocyanate-stained cells treated with IR (4 Gy) or ubenimex ( $\left.0.5 \mathrm{mg} / \mathrm{ml}\right)$ alone or in combination for $24 \mathrm{~h}$ were detected using flow cytometry. "P<0.05 vs. control. Data are presented as the mean $\pm \mathrm{SD}$ of three independent experiments. SD, standard deviation; IR, irradiation; FITC, fluorescein isothiocyanate; PI, propidium iodide; LC3B, microtubule-associated proteins 1A/1B light chain 3B.

examples, autophagy is unequivocally the mode of tumor cell death (23). Although the multiple roles of autophagy in cancer require further clarification, autophagy is directly involved in numerous important physiological processes, including metabolism, response to stress and cell death pathways in cancer cells (38). Both tumor-suppressor genes and oncogenes are implicated in autophagy regulation (39). Accordingly, the role of autophagy in cancer raises a number of questions. Our 

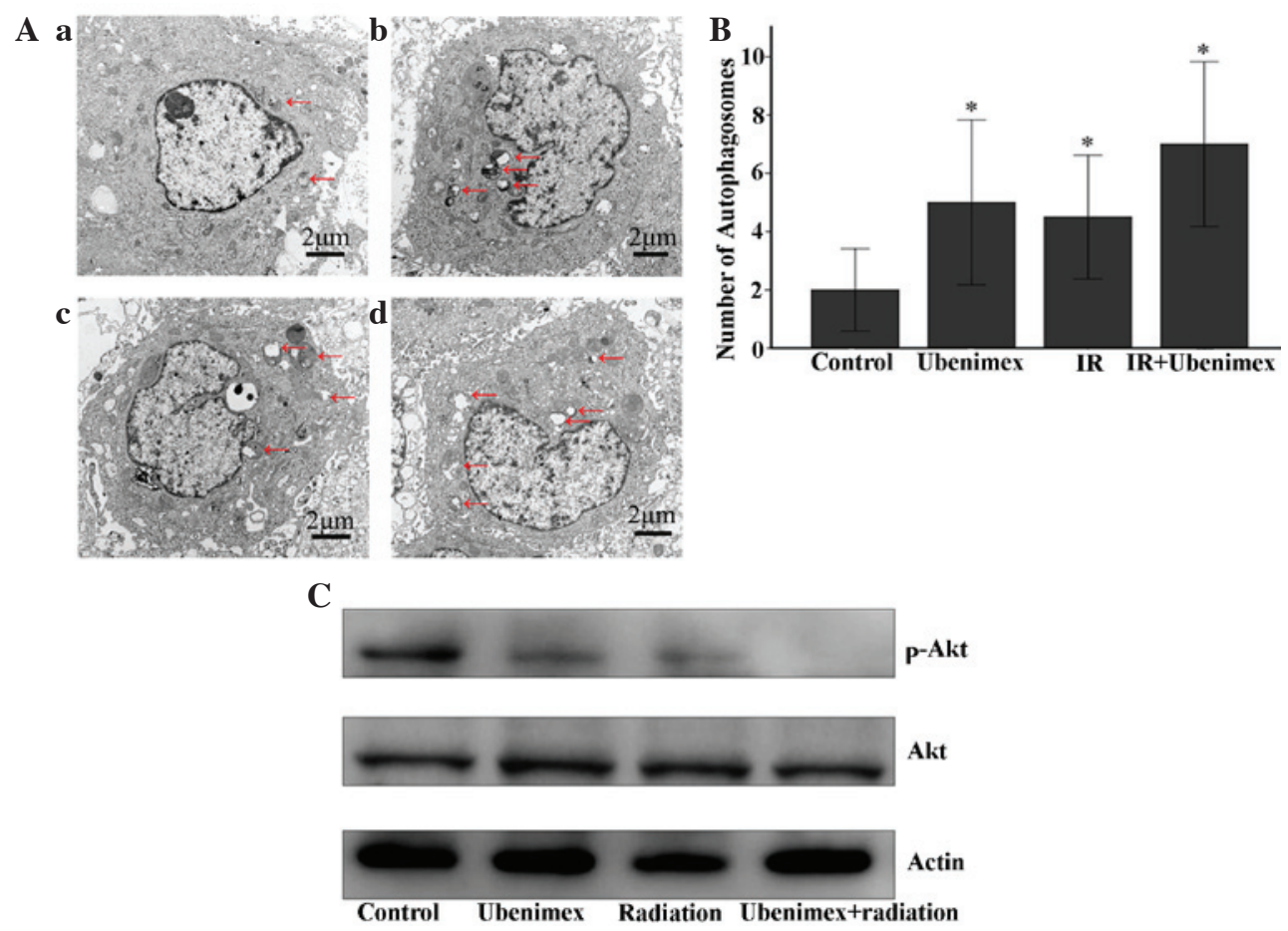

Figure 4. (A) Electron microscopy microphotographs of OS-RC-2 cells treated with IR (4 Gy) or ubenimex $(0.5 \mathrm{mg} / \mathrm{ml})$ alone or in combination for $24 \mathrm{~h}$. (Aa) Control; (Ab) Cells treated with ubenimex $(0.5 \mathrm{mg} / \mathrm{ml})$ alone; (Ac) Cells treated with IR (4 Gy) alone; (Ad) cells treated with combined ubenimex $(0.5 \mathrm{mg} / \mathrm{ml})$ and IR (4 Gy) The red arrows indicate autophagic vacuoles and autolysosomes. Untreated cells were used as a control. (B) Quantification of autophagosomes in 12 random fields in three independent experiments. " $\mathrm{P}<0.05$ vs. control. (C) Western blotting for Akt and p-Akt in OS-RC-2 cells. Cells were treated with IR (4 Gy) or ubenimex $(0.5 \mathrm{mg} / \mathrm{ml})$ alone or in combination for $24 \mathrm{~h}$. Actin served as a loading control. Bars indicate the mean \pm standard deviation from three independent experiments ("P<0.05). IR, irradiation; p-, phosphorylated.

previous study revealed that ubenimex induces autophagy in RCC cells (31). In this context, the present data indicates that the induction of pro-death autophagy increases the radiosensitivity of RCC cells. The possibility that RCC cells may undergo autophagic cell death in response to radiation argues for the potential utility of autophagy as an alternative pathway based on the efficacy of drugs and radiation in promoting tumor cell death.

The Akt signaling pathway plays a crucial role in the regulation of both apoptosis and autophagy (40). The disruption of PI3K/Akt signaling is associated with the induction of autophagy by a variety of antineoplastic agents in cancer cells (40). Viola et al (41) indicated that MG-2477, a tubulin inhibitor, induces autophagy via the inhibition of the Akt signaling pathway in A549 cells. Triptolide induces autophagy in pancreatic cancer cells and also inhibits the Akt pathway (42). The present study demonstrated that the combined treatment of ubenimex and IR significantly decreased the expression of p-Akt in cells compared with ubenimex treatment or IR alone. These results suggest that anticancer agents may commonly induce autophagy by inhibiting Akt. Additionally, previous studies revealed that stress activates the Akt signal transduction pathway in tumor cells, which results in protective autophagy (28). Furthermore, treatment with an Akt inhibitor changed the role of autophagy from protective to lethal (27). These findings suggest that Akt signaling and autophagy are important in the resistance of tumors to treatment. In the present study, treatment with an Akt agonist significantly decreased the autophagic cell death induced by ubenimex as well as radioresistance. This decrease suggests that ubenimex induces Akt-related autophagic cell death. Furthermore, this effect switches the role of radiotherapy-induced autophagy from protective to lethal.

In the present study, ubenimex enhanced the radiosensitivity of RCC cells, and it was demonstrated that the combination of ubenimex and IR modulated the radioresistance of RCC cells. Pretreatment with ubenimex induced pro-death autophagy in the OS-RC-2 and 786-O cell lines in response to radiation. Since ubenimex is well tolerated in clinical adjuvant therapy, it has the potential to be used as a radiosensitizer (28-30). Radiotherapy is not generally considered for the treatment of RCC for a number of reasons, including the relative radioresistance of RCC, the radiosensitivity of the surrounding tissue and the toleration of nephrectomy (31). Importantly, the present results show that ubenimex radiosensitizes RCC, which is essential for the utility of radiotherapy in the treatment of this disease. However, as a novel therapy, ubenimex is unlikely to be tested clinically with radiation without supporting preclinical studies. The present data demonstrate that adding ubenimex increases the effects of clinically relevant doses of radiation in RCC cells.

In summary, the results of the present study demonstrate that the induction of autophagy enhances the radiosensitivity of RCC cells, and that ubenimex switches the role of radiation-induced autophagy from protective to lethal, a switch that is associated with the Akt signaling pathway. In addition, the present findings demonstrate that combining radiotherapy with molecularly targeted therapies is a valid approach for the treatment of RCC that should be further evaluated in preclinical models. Based on these results, ubenimex appears to be an excellent adjunct therapy for the treatment of RCC. Combined 
with rapid advances in both imaging and radiotherapy technologies, adjunct therapy with ubenimex and radiotherapy is an obvious treatment option for RCC in the future.

\section{Acknowledgements}

The present study was funded by grants from the Shandong Provincial Natural Science Foundation (Jinan, China; grant numbers ZR2014HM111 and ZR2014HP015) and the Science and Technology Development Plan Project of Shandong Province, China (grant numbers 2014GGH218036, 2015GSF118055 and 2015GGB14008).

\section{References}

1. Siegel R, Ma J, Zou Z and Jemal A: Cancer statistics, 2014. CA Cancer J Clin 64: 9-29, 2014

2. Hollingsworth JM, Miller DC, Dunn RL, Montgomery JS, Roberts WW, Hafez KS and Wolf JS Jr: Surgical management of low-stage renal cell carcinoma: Technology does not supersede biology. Urology 67: 1175-1180, 2006.

3. Hollingsworth JM, Miller DC, Daignault S and Hollenbeck BK: Rising incidence of small renal masses: A need to reassess treatment effect. J Natl Cancer Inst 98: 1331-1334, 2006.

4. Jin Z and El-Deiry WS: Overview of cell death signaling pathways. Cancer Biol Ther 4: 139-163, 2005.

5. Schmitt CA, Fridman JS, Yang M, Lee S, Baranov E, Hoffman RM and Lowe SW: A senescence program controlled by p53 and p16INK4a contributes to the outcome of cancer therapy. Cell 109: 335-1146, 2002.

6. Chu K, Teele N, Dewey MW, Albright N and Dewey WC: Computerized video time lapse study of cell cycle delay and arrest, mitotic catastrophe, apoptosis and clonogenic survival in irradiated 14-3-3sigma and CDKN1A (p21) knockout cell lines. Radiat Res 162: 270-286, 2004.

7. Ichinose $\mathrm{Y}$, Genka K, Koike T, Kato H, Watanabe Y, Mori T, Iioka S, Sakuma A and Ohta M; NK421 Lung Cancer Surgery Group: Randomized double-blind placebo-controlled trial of bestatin in patients with resected stage I squamous-cell lung carcinoma. J Natl Cancer Inst 95: 605-610, 2003.

8. Wan J, Liu T, Mei L, Li J, Gong K, Yu C and Li W: Synergistic antitumour activity of sorafenib in combination with tetrandrine is mediated by reactive oxygen species (ROS)/Akt signaling. Br J Cancer 109: 342-350, 2013.

9. Nomiya T, Tsuji H, Hirasawa N, Kato H, Kamada T, Mizoe J, Kishi H, Kamura K, Wada H, Nemoto K and Tsujii H: Carbon ion radiation therapy for primary renal cell carcinoma: Initial clinical experience. Int J Radiat Oncol Biol Phys 72: 828-833, 2008.

10. Bursch W: The autophagosomal-lysosomal compartment in programmed cell death. Cell Death Differ 8: 569-581, 2001.

11. Klionsky DJ and Emr SD: Autophagy as a regulated pathway of cellular degradation. Science 290: 1717-1721, 2000.

12. Liu Z, Antalek M, Nguyen L, Li X, Tian X, Le A and Zi X: The effect of gartanin, a naturally occurring xanthone in mangosteen juice, on the mTOR pathway, autophagy, apoptosis, and the growth of human urinary bladder cancer cell lines. Nutr Cancer 65 (Suppl 1): 68-77, 2013.

13. Mizushima N, Tsukamoto S and Kuma A: Autophagy in embryogenesis and cell differentiation. Tanpakushitsu Kakusan Koso 53 (16 Suppl): S2170-S2174, 2008 (In Japanese).

14. Horita H, Frankel AE and Thorburn A: Acute myeloid leukemia-targeted toxin activates both apoptotic and necroptotic death mechanisms. PLoS One 3: e3909, 2008.

15. Ling YH, Aracil M, Zou Y, Yuan Z, Lu B, Jimeno J, Cuervo AM and Perez-Soler R: PM02734 (elisidepsin) induces caspase-independent cell death associated with features of autophagy, inhibition of the AKT/mTOR signaling pathway, and activation of death-associated protein kinase. Clin Cancer Res 17: 5353-5366, 2011.

16. Kim KW, Moretti L, Mitchell LR, Jung DK and Lu B: Combined $\mathrm{Bcl}-2 /$ mammalian target of rapamycin inhibition leads to enhanced radiosensitization via induction of apoptosis and autophagy in non-small cell lung tumor xenograft model. Clin Cancer Res 15: 6096-6105, 2009.
17. Lin CI, Whang EE, Donner DB, Du J, Lorch J, He F, Jiang X, Price BD, Moore FD Jr and Ruan DT: Autophagy induction with RAD001 enhances chemosensitivity and radiosensitivity through Met inhibition in papillary thyroid cancer. Mol Cancer Res 8: 1217-1226, 2010.

18. Chiu HW, Lin JH, Chen YA, Ho SY and Wang YJ: Combination treatment with arsenic trioxide and irradiation enhances cell-killing effects in human fibrosarcoma cells in vitro and in vivo through induction of both autophagy and apoptosis. Autophagy 6: 353-365, 2010.

19. Rajecki M, af Hällström T, Hakkarainen T, Nokisalmi P, Hautaniemi S, Nieminen AI, Tenhunen M, Rantanen V, Desmond RA, Chen DT, et al: Mre11 inhibition by oncolytic adenovirus associates with autophagy and underlies synergy with ionizing radiation. Int J Cancer 125: 2441-2449, 2009.

20. Zhuang W, Li B, Long L, Chen L, Huang Q and Liang Z: Induction of autophagy promotes differentiation of glioma-initiating cells and their radiosensitivity. Int J Cancer 129: 2720-2731, 2011.

21. Altmeyer A, Jung AC, Ignat M, Benzina S, Denis JM, Gueulette J, Noël G, Mutter D and Bischoff P: Pharmacological enhancement of autophagy induced in ahepatocellular carcinoma cell line by high-LET radiation. Anticancer Res 30: 303-310, 2010.

22. Gewirtz DA, Hilliker ML and Wilson EN: Promotion of autophagy as a mechanism for radiation sensitization of breast tumor cells. Radiother Oncol 92: 323-328, 2009.

23. Yang ZJ, Chee CE, Huang S and Sinicrope F: Autophagy modulation for cancer therapy. Cancer Biol Ther 11: 169-176, 2011.

24. Mathew R, Karantza-Wadsworth V and White E: Role of autophagy in cancer. Nat Rev Cancer 7: 961-967, 2007.

25. Rodriguez-Rocha H, Garcia-Garcia A, Panayiotidis MI and Franco R: DNA damage and autophagy. Mutat Res 711: 158-166, 2011.

26. Chen W, Wu J, Shi H, Wang Z, Zhang G, Cao Y, Jiang C and Ding Y: Hepatic stellate cell coculture enables sorafenib resistance in Huh7 cells through HGF/c-Met/Akt and Jak2/Stat3 pathways. Biomed Res Int 2014: 764981, 2014.

27. Zhai B, Hu F, Jiang X, Xu J, Zhao D, Liu B, Pan S, Dong X, Tan G, Wei Z, et al: Inhibition of Akt reverses the acquired resistance to sorafenib by switching protective autophagy to autophagic cell death in hepatocellular carcinoma. Mol Cancer Ther 13: 1589-1598, 2014.

28. Ishii K, Usui S, Sugimura Y, Yoshida S, Hioki T, Tatematsu M, Yamamoto $\mathrm{H}$ and Hirano $\mathrm{K}$ : Aminopeptidase $\mathrm{N}$ regulated by zinc in human prostate participates in tumor cell invasion. Int $\mathrm{J}$ Cancer 92: 49-54, 2001

29. Fontijn D, Duyndam MC, van Berkel MP, Yuana Y, Shapiro LH, Pinedo HM, Broxterman HJ and Boven E: CD13/Aminopeptidase $\mathrm{N}$ overexpression by basic fibroblast growth factor mediates enhanced invasiveness of 1F6 human melanoma cells. Br J Cancer 94: 1627-1636, 2006.

30. Mina-Osorio P: The moonlighting enzyme CD13: Old and new functions to target. Trends Mol Med 14: 361-371, 2008.

31. Liu S, Xie F, Wang H, Liu Z, Liu X, Sun L and Niu Z: Ubenimex inhibits cell proliferation, migration and invasion in renal cell carcinoma: The effect is autophagy-associated. Oncol Rep 33: 1372-1380, 2015.

32. Mujtaba SF, Dwivedi A, Yadav N, Ch R, Kushwaha HN, Mudiam MK, Singh G and Ray RS: Superoxide mediated photomodification and DNA damage induced apoptosis by Benz(a) anthracene via mitochondrial mediated pathway. J Photochem Photobiol B 142: 92-102, 2015.

33. Liu K, Liu PC, Liu R and Wu X: Dual AO/EB staining to detect apoptosis in osteosarcoma cells compared with flow cytometry. Med Sci Monit Basic Res 21: 15-20, 2015

34. Zhang S, Qi J, Sun L, Cheng B, Pan S, Zhou M and Sun X: Matrine induces programmed cell death and regulates expression of relevant genes based on PCR array analysis in C6 glioma cells. Mol Biol Rep 36: 791-799, 2009.

35. Burdak-Rothkamm S and Prise KM: New molecular targets in radiotherapy: DNA damage signalling and repair in targeted and non-targeted cells. Eur J Pharmacol 625: 151-155, 2009.

36. Elmore S: Apoptosis: A review of programmed cell death. Toxicol Pathol 35: 495-516, 2007.

37. Anbalagan S, Pires IM, Blick C, Hill MA, Ferguson DJ, Chan DA and Hammond EM: Radiosensitization of renal cell carcinoma in vitro through the induction of autophagy. Radiother Oncol 103: 388-393. 2012.

38. Rosenfeldt MT and Ryan KM: The multiple roles of autophagy in cancer. Carcinogenesis 32: 955-963, 2011. 
39. Morani F, Titone R, Pagano L, Galetto A, Alabiso O, Aimaretti $\mathrm{G}$ and Isidoro $\mathrm{C}$ : Autophagy and thyroid carcinogenesis: Genetic and epigenetic links. Endocr Relat Cancer 21: R13-R29, 2013.

40. Takeuchi H, Kondo Y, Fujiwara K, Kanzawa T, Aoki H, Mills GB and Kondo S: Synergistic augmentation of rapamycin-induced autophagy in malignant glioma cells by phosphatidylinositol 3-kinase/protein kinase B inhibitors. Cancer Res 65 3336-3346, 2005.
41. Viola G, Bortolozzi R, Hamel E, Moro S, Brun P, Castagliuolo I, Ferlin MG and Basso G: MG-2477, a new tubulin inhibitor, induces autophagy through inhibition of the Akt/mTOR pathway and delayed apoptosis in A549 cells. Biochem Pharmacol 83: 16-26, 2012.

42. Mujumdar N, Mackenzie TN, Dudeja V, Chugh R, Antonoff MB, Borja-Cacho D, Sangwan V, Dawra R, Vickers SM and Saluja AK: Triptolide induces cell death in pancreatic cancer cells by apoptotic and autophagic pathways. Gastroenterology 139: 598-608, 2010. 